\title{
Two-component model for the interstellar scattering in direction to the Vela pulsar
}

\author{
A. Z. Zhou ${ }^{1}$, A. Esamdin ${ }^{2}$, and X. J. Wu ${ }^{2,3}$ \\ 1 Shanghai Astronomical Observatory, Chinese Academy of Sciences, Shanghai 200030, PR China \\ e-mail: zaiz@center.shao.ac.cn \\ 2 Urumqi Station, the National Astronomical Observatories, the Chinese Academy of Sciences, Urumqi 830011, PR China \\ 3 Department of Astronomy, Peking University, Beijing 100871, PR China
}

Received 30 December 2004 / Accepted 31 March 2005

\begin{abstract}
This paper discusses a rigorous treatment of the refractive scintillation of pulsar PSR B0833-45 caused by a twocomponent interstellar scattering medium. It is assumed that the interstellar scattering medium is composed of a thin screen ISM and an extended interstellar medium. We consider that the scattering of the thin screen concentrates in a thin layer presented by a $\delta$ function distribution and that the scattering density of the extended irregular medium satisfies the Gaussian distribution. We investigate and develop equations for the flux density structure function corresponding to this two-component ISM geometry in the scattering density distribution and compare our result with that of the Vela pulsar observations. We conclude that the refractive scintillation caused by this two-component ISM scattering gives a more satisfactory explanation for the observed flux density variation of the Vela pulsar than does the single extended medium model. The level of refractive scintillation is strongly sensitive to the distribution of scattering material along the line of sight. The logarithmic slope of the structure function is sensitive to thin screen location and is relatively insensitive to the scattering strength of the thin screen medium. Therefore, the proposed model can be applied to interpret the structure function of flux density observed in pulsar PSR B0833-45. The result suggests that the medium consists of a discontinuous distribution of plasma turbulence embedded in the Vela supernova remnant. Thus our work provides some insight into the distribution of the scattering along the line of sight to the Vela pulsar.
\end{abstract}

Key words. stars: pulsars: individual: PSR B0833-45 - radio continuum: stars - ISM: structure - scattering

\section{Introduction}

Pulsars are excellent probes of the interstellar medium (ISM). Observed pulse profiles are influenced by dispersion, scattering, and Faraday rotation along the line of sight (LOS) from the Earth to the pulsar. In the strong scattering region, two kinds of propagation effects, diffractive and refractive interstellar scintillation (DISS and RISS), have been recognized and are now thought to be responsible for many effects seen in pulsars (Hewish et al. 1968; Cordes et al. 1986; Rickett et al. 1984, 1990; Gupta et al. 1993, 1994; Armstrong et al. 1995).

Although several authors have suggested that the observed variation in pulsar flux densities at radio frequencies is caused by either intrinsic luminosity fluctuations or propagation effects, or even by some combination of both (Stinebring \& Condon 1990; Kaspi \& Stinebring 1992; Wu \& Chian 1995), the long-term flux density variations with the characteristic timescale of days to months are caused by RISS effects (Sieber 1982; Rickett et al. 1984; Rickett 1990).

As for the nature and distribution of the interstellar medium (ISM), there have been two classical theoretical models dealing with distribution of the ISM scattering density.
Blandford \& Narayan (1985) introduce a simple thin "equivalent screen" of scattering material model to compute fluctuations in mean intensity, image size, and pulse width. In later work, Romani et al. (1986) test the validity of the thin-screen approximation and developed a formalism to treat scattering in an extended medium. Furthermore, Blandford et al. (1986) regard the galactic scattering medium as a Gaussian disk and the scattering strength to be consistent with a Gaussian distribution. In fact, the distribution of scattering material in the local ISM is not uniform and a local bubble may be surrounded by a shell of higher density fluctuations (Bhat et al. 1998). Bhat et al. (1999b) discuss many different models to constrain the electron density spectrum in the local ISM. Several authors have measured the pulse broadening caused by multipath propagation through the interstellar medium to investigate the LOS ISM effects and to model the ionized content of the ISM (Bhat et al. 2004; Taylor \& Cordes 1993, hereafter TC93; Bhat \& Gupta 2002; Cordes \& Lazio 2002, 2003). Other authors have considered the flux density variations (Rickett \& Lyne 1990; LaBrecque et al. 1994; Esamdin et al. 2000, 2004) and the measurement of DISS parameters to derive the scattering strength and scintillation speeds (Gupta et al. 1994; 
Stinebring et al. 1996; Bhat et al. 1999a; Wang et al. 2001). Recently, Stinebring et al. (2000, hereafter S2000) monitored the radio flux density of 21 pulsars at $610 \mathrm{MHZ}$ for five years and presented the structure functions of flux density time series.

Descriptions in terms of structure functions of observable quantities are extensively used to analyze the flux density time series. The slope of the structure function, $\gamma$, probes both the spectrum of the ionized medium and its distribution along the line of sight. For scattering material confined to a thin screen and obeying a Kolmogorov spectrum, the expected value is $\gamma=2.0$ (Coles 1988; Romani et al. 1986). By contrast, the expected value of the logarithmic slope for a Kolmogorov spectrum and an extended distribution of scattering material is $\gamma=\beta-3$ (Coles 1988; Shishov 1993). However, this conclusion is highly sensitive to the detailed distribution of the scattering material (Cordes \& Rickett 1998). Stinebring et al. (2000) show that the logarithmic slope for two-thirds of the 21 pulsars considered is in the range $0.4-1.0$; about one-third are clustered at higher values 1.4-1.6, of which the structure function of the flux density of thirteen pulsars have a single slope. In our previous work (Zhou et al. 2003a,b,c), we fitted the structure function of the flux density for seven pulsars using the single extended ISM model. However, for the special Vela pulsar, the observed logarithmic slope of the flux density is 0.9. Obviously, this kind of structure function of flux density can't be interpreted by the single simple thin screen ISM model or the single extended ISM model. In fact, the free electron density distribution in the ISM can be decomposed into three different regimes: the spiral arms consisting of gaint HII regions, the inner disk comprising a dense ionized region, and the thick disk filled with diffuse electron gas (TC93). For simplicity, the asymmetric interstellar medium is commonly made up of two components: the extended scattering medium and the local scattering medium, a dense nebula (Cordes et al. 1991).

In this paper a two-component ISM model is proposed to interpret the flux density structure function of the Vela pulsar and compared with the observational results of S2000. Our work can serve as a useful probe for understanding the spatial distribution of scattering material along the LOS. The paper is organized as follows. The proposed two-component ISM model is described in detail in Sect. 2. The fitted results and the comparison with the observation are presented in Sect. 3, and in the last section we summarize our conclusions and discussion.

\section{The two-component ISM model}

The RISS theory is often used to calculate the structure function of flux density (Romani et al. 1986; Blandford et al. 1986), which assumes that the spectrum of the electron density fluctuations follows an isotropic power-law:

$p(\kappa)=C_{N}^{2}(z) \kappa^{-\beta}$ for $\kappa_{\text {out }} \leq \kappa \leq \kappa_{\text {inn }}$,

where $\kappa=2 \pi / s$ is the spatial wave number of the fluctuation, $s$ the spatial scale, and $\kappa_{\text {inn }}$ and $\kappa_{\text {out }}$ are the inner and outer cutoffs of scale size, respectively. The indicator of rms electron density fluctuations, $C_{N}^{2}$, characterizes the strength of the scattering and is a function of location $z$ in the Galaxy. The value

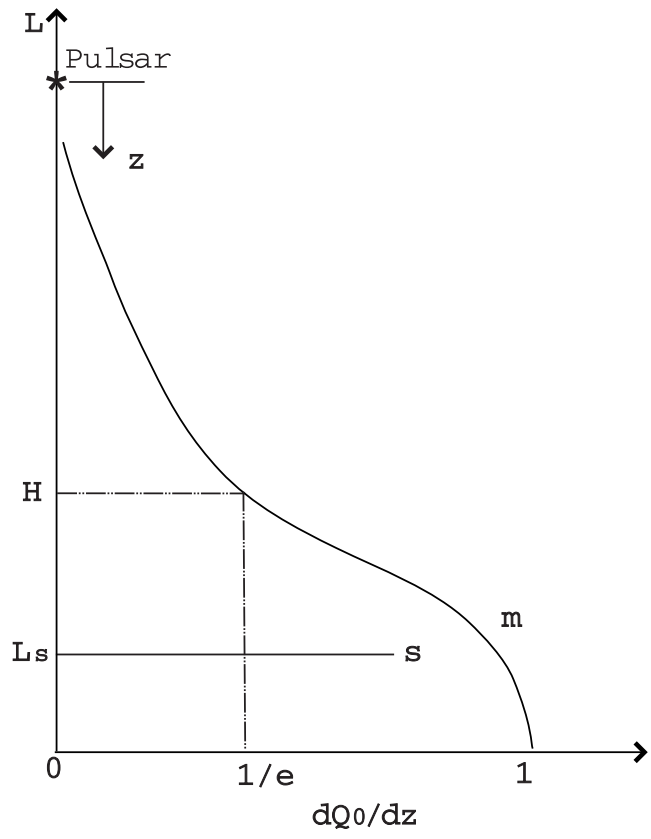

Fig. 1. Scattering density distribution of ISM (m-extended medium, s-thin screen medium).

of the power-law index $\beta$, which is in the range $3<\beta<5$, determines whether the scattering is thought of as a turbulence spectrum $(\beta<4)$ or a stochastic superposition of discrete scattering structures $(\beta>4)$. Kolmogorov turbulence of the index $\beta=11 / 3$ is a model that is often discussed in the literature.

Blandford et al. (1986) assume that the scattering density distribution of the extended medium is a Gaussian; we assume, however, that the total scattering strength density distribution of the two-component interstellar medium is the sum of a thinscreen and extended medium, namely:

$\frac{\mathrm{d} Q_{0}(z)}{\mathrm{d} z}=\frac{\mathrm{d} Q_{\mathrm{s}}(z)}{\mathrm{d} z}+\frac{\mathrm{d} Q_{\mathrm{m}}(z)}{\mathrm{d} z}=Q_{\mathrm{s} 0} \delta\left(z-z_{\mathrm{s}}\right)+\frac{2 Q_{\mathrm{m} 0}}{\sqrt{\pi} H} \exp \left(-\frac{L^{2}}{H^{2}}\right)$,

where the subscripts " $\mathrm{s}$ " and " $\mathrm{m}$ " represent the thin screen medium and the extended medium, respectively. The scattering strength of the thin screen medium is presented by a $\delta$ function distribution, while the scattering density of the extended medium is consistent with a Gaussian distribution. $Q_{\mathrm{s} 0}$ and $Q_{\mathrm{m} 0}$ are the total scattering strength of the thin screen and extended medium, respectively, $H$ is the $1 /$ e width of the distribution, $L$ is the distance to the observer, and $z_{\mathrm{s}}$ is the distance from the pulsar to the thin screen. Apparently from Fig. $1, L=D-z$ and $L_{\mathrm{s}}=D-z_{\mathrm{s}}$. Similar to the adopted figure in other authors' work (Qian \& Zhang 1996), there are two free distance parameters used here, $z$ and $L$, going in opposite directions for their convenience in the following mathematical description and derivation of equations.

The autocorrelation function $F(\tau)$ and the structure function $D(\tau)$ can be written as:

$$
\begin{aligned}
F(\tau)= & \langle\delta F(t) \delta F(t+\tau)\rangle_{t}, \\
D(\tau)= & 2 \times[F(0)-F(\tau)]=2 \times\left[\langle\delta F(t) \delta F(t)\rangle_{t}\right. \\
& \left.-\langle\delta F(t) \delta F(t+\tau)\rangle_{t}\right]
\end{aligned}
$$


where $\delta F(t)$ represents the normalized flux variation, defined as $\delta F(t)=\left[F(t)-\langle F\rangle_{t}\right] /\langle F\rangle_{t}$ (Hjellming \& Narayan 1986), $F(t)$ denotes the measured flux at time $t$ for some frequency, $\tau$ is the time lag and the subscript $t$ means averaging over all $t$ s.

The autocorrelation function of the flux density is then given by (For simplicity we assume that the power-law index of the electron density fluctuation spectrum is given by $\beta=4$ ) (Romani et al. 1986):

$$
\begin{aligned}
F(\tau)= & \frac{\lambda^{4}}{(2 \pi)^{5} D^{2}} \int_{0}^{D} \mathrm{~d} z(D-z)^{2} z^{2} \frac{1}{s^{2}(z)} \exp \left[-\frac{V^{2} \tau^{2}}{2 s^{2}}\left(\frac{z}{d}\right)^{2}\right] \\
& \times \frac{\mathrm{d} Q_{0}(z)}{\mathrm{d} z},
\end{aligned}
$$

where $D$ is the distance of the source, $V$ the transverse velocity of the source relative to the observer, $\lambda$ the observation wavelength, and $s(z)(s(z)=L \theta(z))$ and $\theta(z)$ are the apparent linear radius and angular radius of the source at the distance $L$, respectively. $s^{2}(z)$ is given by:

$$
\begin{aligned}
s^{2}(z)= & L^{2} \theta^{2}(z)=\left(\frac{D-z}{D}\right)^{2} \int_{0}^{z} \mathrm{~d} z^{\prime} z^{\prime 2} \psi\left(z^{\prime}\right) \\
& +\frac{z^{2}}{D^{2}} \int_{z}^{\infty} \mathrm{d} z^{\prime}\left(D-z^{\prime}\right)^{2} \psi\left(z^{\prime}\right),
\end{aligned}
$$

where $\psi(z)$ is defined as the average scattering probability per unit length:

$\psi(z)=\left\langle\frac{\Delta \theta^{2}(z)}{\Delta z}\right\rangle$.

For the proposed two-component ISM model,

$\psi(z)=\psi_{\mathrm{s}}(z)+\psi_{\mathrm{m}}(z)$,

with

$\psi_{\mathrm{s}}(z)=\theta_{\mathrm{s} 0}^{2} \delta\left(z-z_{\mathrm{s}}\right)$,

where $\theta_{\mathrm{s} 0}$ is the total scattering angle width of the thin screen medium:

$\theta_{\mathrm{s} 0}^{2}=(1.06)^{2}\left(\frac{\lambda}{2 \pi}\right)^{4} \gamma Q_{\mathrm{s} 0} \quad$ (cgs - units $)$.

While the average scattering probability of the extended medium is

$\psi_{\mathrm{m}}(z)=\theta_{\mathrm{m} 0}^{2} \frac{2}{\sqrt{\pi} H} \exp \left[-\left(\frac{D-z}{H}\right)^{2}\right]$,

where $\theta_{\mathrm{m} 0}$ is the total scattering angle width of the extended medium:

$\theta_{\mathrm{m} 0}^{2}=(1.06)^{2}\left(\frac{\lambda}{2 \pi}\right)^{4} \gamma Q_{\mathrm{m} 0} \quad$ (cgs - units $)$

where the factor $\gamma$ is unity and has only a weak logarithmic dependence on $C_{-4}, \lambda$ and $D$ (Romani et al. 1986).

For the autocorrelation function if we define $y=\frac{L}{H}$ and $y_{\mathrm{s}}=\frac{L_{\mathrm{s}}}{H}$ we obtain

$$
\begin{aligned}
F(\tau)= & \frac{\lambda^{4}}{(2 \pi)^{5}} \frac{Q_{\mathrm{s} 0}}{\theta^{2}\left(z_{\mathrm{s}}\right)} \exp \left[-\frac{v^{2} \tau^{2}}{2 L_{\mathrm{s}}^{2} \theta^{2}\left(z_{\mathrm{s}}\right)}\right] \\
& +\frac{Q_{\mathrm{m} 0}}{\pi^{3 / 2}}\left(\frac{\lambda}{2 \pi}\right)^{4} \int_{0}^{D} \frac{\mathrm{e}^{-y^{2}}}{\theta^{2}(y)} \exp \left[-\frac{v^{2} \tau^{2}}{2 H^{2} y^{2} \theta^{2}(y)}\right] \mathrm{d} y
\end{aligned}
$$

where

$\theta^{2}\left(z_{\mathrm{s}}\right)=\theta^{2}\left(L_{\mathrm{s}}\right)=\theta_{\mathrm{s} 0}^{2}+\theta_{\mathrm{m} 0}^{2}\left[1-\frac{2}{\sqrt{\pi}} \int_{0}^{y_{\mathrm{s}}}\left(1-\frac{x^{2}}{y^{2}}\right) \mathrm{e}^{-x^{2}} \mathrm{~d} x\right]$

$\theta^{2}(y)=\theta_{\mathrm{s} 0}^{2}+\theta_{\mathrm{m}}^{2}(y)\left(\right.$ for $\left.y<y_{\mathrm{s}}\right)$

$\theta^{2}(y)=\frac{L_{\mathrm{s}}^{2} \theta_{\mathrm{s} 0}^{2}}{L^{2}}+\theta_{\mathrm{m}}^{2}(y)\left(\right.$ for $\left.y>y_{\mathrm{s}}\right)$

$\theta_{\mathrm{m}}^{2}(y)=\theta_{\mathrm{m} 0}^{2}\left[1-\frac{2}{\sqrt{\pi}} \int_{0}^{y}\left(1-\frac{x^{2}}{y^{2}}\right) \mathrm{e}^{-x^{2}} \mathrm{~d} x\right]$.

In Eqs. (15) and (16) we take into account the differing influence of the interstellar medium on the scattering angle on either side of the thin screen, i.e. in Eq. (15) $\theta_{\mathrm{s} 0}^{2}$ and in Eq. (16) we use $\left(\frac{L_{\mathrm{s}}}{L}\right)^{2}$ as a weighting factor.

Then the autocorrelation function is obtained (with $d_{\mathrm{H}}=$ $\left.\frac{D}{H}\right)$ :

$$
\begin{aligned}
F(\tau)= & \frac{Q_{\mathrm{m} 0}\left(\frac{\lambda}{2 \pi}\right)^{4}}{\pi^{3 / 2}} \int_{0}^{y_{\mathrm{s}}} \mathrm{e}^{-y^{2}}\left(1-\frac{y}{d_{\mathrm{H}}}\right)^{2}\left(p[y]+\theta_{\mathrm{s}}^{2}\right)^{-1} \\
& \times \exp \left[\frac{-v^{2} \tau^{2}\left(1-\frac{y}{d_{\mathrm{H}}}\right)^{2}\left(p[y]+\theta_{\mathrm{s}}^{2}\right)^{-1}}{2 H^{2} y^{2}}\right] \mathrm{d} y \\
& +\frac{Q_{\mathrm{m} 0}\left(\frac{\lambda}{2 \pi}\right)^{4}}{\pi^{3 / 2} \int_{y_{\mathrm{s}}}^{d_{\mathrm{H}}} \mathrm{e}^{-y^{2}}\left(1-\frac{y}{d_{\mathrm{H}}}\right)^{2}\left(p[y]+\frac{y_{\mathrm{s}}^{2} \theta_{\mathrm{s}}^{2}}{y^{2}}\right)^{-1}} \\
& \times \exp \left[\frac{-v^{2} \tau^{2}\left(1-\frac{y}{d_{\mathrm{H}}}\right)^{2}\left(p[y]+\frac{y_{\mathrm{s}}^{2} \theta_{\mathrm{s}}^{2}}{y^{2}}\right)^{-1}}{2 H^{2} y^{2}}\right] \mathrm{d} y \\
& +\frac{Q_{\mathrm{s} 0}\left(\frac{\lambda}{2 \pi}\right)^{4}}{2 \pi}\left(1-\frac{L_{\mathrm{s}}}{d}\right)^{2}\left(p\left[y_{\mathrm{s}}\right]+\theta_{\mathrm{s}}^{2}\right)^{-1} \\
& \times \exp \left[\frac{-v^{2} \tau^{2}\left(1-\frac{L_{\mathrm{s}}}{D}\right)^{2}\left(p\left[y_{\mathrm{s}}\right]+\theta_{\mathrm{s}}^{2}\right)^{-1}}{2 L_{\mathrm{s}}^{2}}\right]
\end{aligned}
$$

So the structure function of the flux density for the twocomponent ISM model is as follows:

$$
\begin{aligned}
D(\tau)= & 2 \frac{Q_{\mathrm{m} 0}\left(\frac{\lambda}{2 \pi}\right)^{4}}{\pi^{3 / 2}} \int_{0}^{y_{\mathrm{s}}} \mathrm{e}^{-y^{2}}\left(1-\frac{y}{d_{\mathrm{H}}}\right)^{2}\left(p[y]+\theta_{\mathrm{s}}^{2}\right)^{-1} \\
& \times\left(1-\exp \left[\frac{-v^{2} \tau^{2}\left(1-\frac{y}{d_{\mathrm{H}}}\right)^{2}\left(p[y]+\theta_{\mathrm{s}}^{2}\right)^{-1}}{2 H^{2} y^{2}}\right]\right) \mathrm{d} y \\
& +2 \frac{Q_{\mathrm{m} 0}\left(\frac{\lambda}{2 \pi}\right)^{4}}{\pi^{3 / 2}} \int_{y_{\mathrm{s}}}^{d_{\mathrm{H}}} \mathrm{e}^{-y^{2}}\left(1-\frac{y}{d_{\mathrm{H}}}\right)^{2}\left(p[y]+\frac{y_{\mathrm{s}}^{2} \theta_{\mathrm{s}}^{2}}{y^{2}}\right)^{-1} \\
& \times\left(1-\exp \left[\frac{\left.\left.-v^{2} \tau^{2}\left(1-\frac{y}{d_{\mathrm{H}}}\right)^{2}\left(p[y]+\frac{y_{\mathrm{s}}^{2} \theta_{\mathrm{s}}^{2}}{y^{2}}\right)^{-1}\right]\right) \mathrm{d} y}{2 H^{2} y^{2}}\right]\right) \\
& +2 \frac{Q_{\mathrm{s} 0}\left(\frac{\lambda}{2 \pi}\right)^{4}}{2 \pi}\left(1-\frac{L_{\mathrm{s}}}{d}\right)^{2}\left(p\left[y_{\mathrm{s}}\right]+\theta_{\mathrm{s}}^{2}\right)^{-1} \\
& \times\left(1-\exp \left[\frac{-v^{2} \tau^{2}\left(1-\frac{L_{\mathrm{s}}}{D}\right)^{2}\left(p\left[y_{\mathrm{s}}\right]+\theta_{\mathrm{s}}^{2}\right)^{-1}}{2 L_{\mathrm{s}}^{2}}\right]\right)
\end{aligned}
$$


Table 1. Best fit parameters for the two-component model and comparison with observational results from S2000 for PSR B0833-45.

\begin{tabular}{cccccccccc}
\hline \hline$\beta$ & $\begin{array}{c}D \\
(\mathrm{kpc})\end{array}$ & $\begin{array}{c}H \\
(\mathrm{kpc})\end{array}$ & $\begin{array}{c}V_{\mathrm{t}} \\
\left(\mathrm{km} \mathrm{s}^{-1}\right)\end{array}$ & $\begin{array}{c}V_{\mathrm{pm}} \\
\left(\mathrm{km} \mathrm{s}^{-1}\right)\end{array}$ & $\begin{array}{c}C_{-4, \mathrm{~m}} \\
\left(\mathrm{~m}^{-20 / 3}\right)\end{array}$ & $\begin{array}{c}C_{-4, \mathrm{~s}} \\
\left(\mathrm{~m}^{-20 / 3}\right)\end{array}$ & $\begin{array}{c}C_{-4, \text { DISS }} \\
\left(\mathrm{m}^{-20 / 3}\right)\end{array}$ & $\begin{array}{c}L_{\mathrm{s}} \\
(\mathrm{kpc})\end{array}$ & $\begin{array}{c}\text { The } \\
\text { model }\end{array}$ \\
\hline 4 & 0.5 & 0.25 & 120 & 120 & $10^{4.3}$ & $10^{4.2}$ & $10^{4.3}$ & 0.4 & $\mathrm{~T}$ \\
$11 / 3$ & 0.5 & 0.25 & 120 & 120 & $10^{4.3}$ & - & $10^{4.3}$ & - & $\mathrm{S}$ \\
4 & 0.5 & 0.25 & 120 & 120 & $10^{4.3}$ & - & $10^{4.3}$ & - & $\mathrm{S}$ \\
\hline
\end{tabular}

where

$$
\begin{aligned}
p[y]= & \theta_{\mathrm{m}}^{2} \frac{2}{\sqrt{\pi}} \int_{0}^{d_{\mathrm{H}}} \mathrm{e}^{-\omega^{2}}\left(1-\frac{\omega}{d_{\mathrm{H}}}\right)^{2} \mathrm{~d} y \\
& -\int_{0}^{y}\left[\mathrm{e}^{-\omega^{2}}\left(1-\frac{\omega}{d_{\mathrm{H}}}\right)^{2}-\left(1-\frac{y}{d_{\mathrm{H}}}\right)^{2} \times \frac{\omega^{2}}{y^{2}}\right] \mathrm{d} y,
\end{aligned}
$$

and,

$$
\begin{aligned}
& Q_{\mathrm{m} 0}=1.6 * 10^{-21} * D_{\mathrm{kpc}} C_{-4, \mathrm{~m}}\left(\mathrm{~cm}^{-11 / 3}\right) \\
& Q_{\mathrm{s} 0}=1.6 * 10^{-21} * D_{\mathrm{kpc}} C_{-4, \mathrm{~s}}\left(\mathrm{~cm}^{-11 / 3}\right) \\
& \theta_{\mathrm{m}}=2.2 * D_{\mathrm{kpc}}^{0.5} \lambda_{\mathrm{m}}^{2} C_{-4, \mathrm{~m}}^{0.5} \text { (mas) } \\
& \theta_{\mathrm{s}}=2.2 * D_{\mathrm{kpc}}^{0.5} \lambda_{\mathrm{m}}^{2} C_{-4, \mathrm{~s}}^{0.5} \text { (mas) }
\end{aligned}
$$

where $D$ and $\lambda$ are, respectively, in units of $\mathrm{kpc}$ and $\mathrm{m}$ and $C_{-4}=\overline{C_{N}^{2}} / 10^{-4}$. There are six parameters in Eqs. (19)-(24): $D$, $\mathrm{V}, \mathrm{H} C_{-4, \mathrm{~m}}, C_{-4, \mathrm{~s}}, L_{\mathrm{s}}$.

The pulsar distance $0.5 \mathrm{kpc}$ is derived from the TC93 model of the Galactic electron density distribution, and the proper motion velocity $180 \mathrm{~km} \mathrm{~s}^{-1}$ is given by Lyne et al. (1982). As Bhat et al. (1999a) mention, the scattering screen of the extended ISM is usually placed midway between the observer and the pulsar; therefore we adopt $H=0.25 \mathrm{kpc}$. The other three parameters $C_{-4, \mathrm{~m}}, C_{-4, \mathrm{~s}}$, and $L_{\mathrm{s}}$ are taken as free parameters in the calculation.

The best sets of the parameter values used to calculate the theoretical flux density structure function and some of the observational ones from S2000 for comparison are listed in Table 1. Columns 1-3 give the power-law index, the pulsar distance, and the $1 / e$ width of the scattering strength of the extended medium. The best-fitted velocity $V_{\mathrm{t}}$ and the proper motion velocity $V_{\mathrm{pm}}$ are listed in Cols. 4 and 5 for comparison. $C_{-4, \mathrm{~m}}$ and $C_{-4, \mathrm{~s}}$ are the scattering strength of the extended medium and thin screen medium, respectively, shown in Cols. 6 and 7 . The observed values of the scattering strength (calculated by $C_{-4}=\overline{C_{N}^{2}} / 10^{-4}$ ) from DISS measurements (S2000) are listed in Col 8. In Col. 9, the distance from the observer to the thin screen $L_{\mathrm{S}}$ is presented. Column 10 indicates the different ISM models adopted for the fit procedure. The subscripts " $\mathrm{S}$ " and "T" represent the single extended ISM model and the two-component ISM model, respectively.

Referring to the location of the Vela Nebula, the Vela supernova remnant has been shown to be embedded in a hot bubble confined by the shell of the Gum Nebula (Aschenbach et al. 1995). Older literature suggests a distance of 500 pc (e.g. Cha et al. 1999); this value has been revised recently by Caraveo et al. (2001) to $\sim 300$ pc. Our fitted results suggest that the Vela supernova remnant, assumed as a thin screen medium, is located near the pulsar with a distance $0.4 \mathrm{kpc}$ from the observer to the thin screen.

The fitted scattering strength value of the extended medium $10^{4.3} \mathrm{~m}^{-20 / 3}$ equals the observed value (S2000), while the fitted scattering strength value of the thin screen is $10^{4.2} \mathrm{~m}^{-20 / 3}$, smaller than that of the extended medium. This may indicate that a denser region exists with enhanced scattering along the LOS and concentrated in the Vela nebula, and that the scattering of the thin screen dominates.

\section{The fitted results}

In Fig. 2 we plotted the structure function versus time lag as observed for the Vela pulsar. Figure 2a shows the theoretical curves for the "two-component" scattering model with various scattering strength values of the thin screen medium, and Fig. $2 b$ does the same with various values of the distance from the observer to the thin screen. The slope of the structure function is independent of the scattering strength of the thin screen, but depends somewhat on the location of the thin screen, or on the value of $L_{\mathrm{s}}$ that largely parameterizes the "two-component" model in contrast to the single-extended medium model. By contrast, theoretical curves are also shown in Fig. 3 for the single-extended medium geometry. The dashed and dotted lines correspond to the Kolmogorov spectrum $(\beta=$ $11 / 3$ ) and the $\beta=4$ spectrum for the extended medium model, respectively. The theoretical result from the "two-component" model, indicated by the solid line, shows stronger consistency with the observations than is predicted by both the Kolmogorov and the $\beta=4$ model for the single-extended medium. Thus it can be concluded that along the LOS to the Vela pulsar, the spectrum of the electron density fluctuations corresponds to the super-Kolmogorov form, which is in good agreement with the conclusions of S2000.

There are three important quantities of the structure function which characterize the properties of pulsar flux density variation: $m, \tau_{\mathrm{r}}$, and $\gamma$. The modulation index $m$ denotes the amplitude of the flux density fluctuations and is defined as $m=\sqrt{D_{\infty} / 2}$, where $D_{\infty}$ is the saturation value. $\tau_{\mathrm{r}}$ is the value of the scintillation timescale, the time lag at which the structure function reaches half its saturation value. $\gamma$ is the logarithmic slope of the structure function, which is related to the spectrum of electron density fluctuations in the ISM and the distribution along the line of sight. The relation $D(\tau) \propto \tau^{\gamma}$ is used to calculate $\gamma$. 

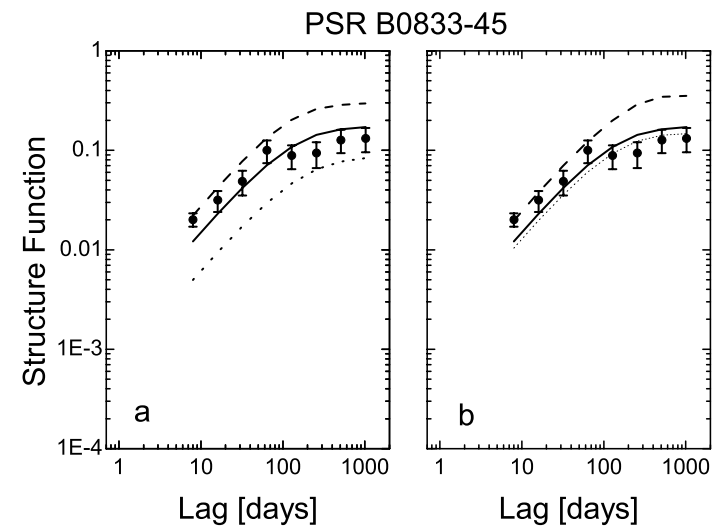

Fig. 2. Theoretical structure functions and observations of PSR B0833-45. a) $C_{-4, \mathrm{~s}}$ is treated as a free parameter: the dashed, solid, and dotted lines represent $C_{-4, \mathrm{~s}}=10^{3.8}, 10^{4.2}$, and $10^{4.6} \mathrm{~m}^{-20 / 3}$, respectively. b) $L_{\mathrm{s}}$ is treated as a free parameter: the dashed, solid, and dotted lines represent $L_{\mathrm{s}}=0.2,0.4$, and $0.48 \mathrm{kpc}$, respectively. Note: Solid dots are observational data from S2000. Solid lines represent theoretical results by the adoption of the best parameters listed in line 1 of Table 1. Dashed and dotted lines show the influence of variation in one parameter on the structure function, while all the other parameters were kept constant in accordance with Table 1.

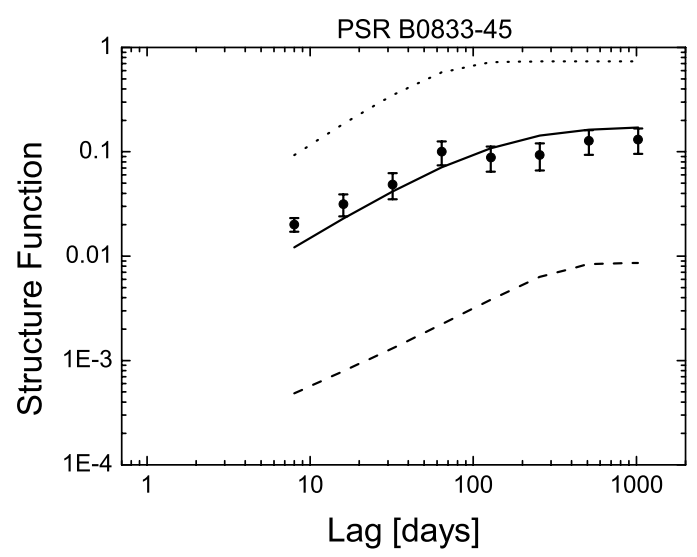

Fig. 3. Theoretical structure functions and observations of PSR B0833-45. The solid line represents the two-component model of the ISM, and the dashed and dotted lines the single extended medium model with $\beta=11 / 3$ and $\beta=4$, respectively.

The theoretical and observational values of $m, \tau_{\mathrm{r}}$, and $\gamma$ are listed in Table 2, where subscripts " $t$ " and "o" represent the theoretical and the observational values, respectively. It appears that, although the theoretical value of the timescale (65 days) is slightly higher than the observed value (35 days), the theoretical value of the modulation index and logarithmic slope are both consistent with the observational values.

\section{Conclusions and discussions}

In this paper a two-component ISM scattering model for discontinuous density structures of a $\beta=4$ electron density spectrum was derived and compared with observations of the Vela pulsar flux density structure function. This at first seems a promising explanation for the flux density variation observed
Table 2. Observed and predicted RISS parameters.

\begin{tabular}{cccccccc}
\hline \hline \multirow{2}{*}{ PSR B } & & \multicolumn{3}{c}{ Observed parameters } & \multicolumn{3}{c}{ Predicted parameters } \\
\cline { 3 - 8 } & $v_{\mathrm{obs}}$ & $m_{\mathrm{r}}$ & $\begin{array}{c}T_{\mathrm{r}} \\
\text { (days) }\end{array}$ & $\gamma$ & $m_{\mathrm{r}, \mathrm{p}}$ & $\begin{array}{c}T_{\mathrm{r}, \mathrm{p}} \\
\text { (days) }\end{array}$ & $\gamma_{\mathrm{p}}$ \\
\hline $0833-45$ & 610 & $0.24 \pm 0.03$ & 35 & 0.9 & 0.28 & 65 & 0.8 \\
\hline
\end{tabular}

along the line of sight to the Vela pulsar due to the refractive scintillation.

From Fig. 3, we find that the two-component model provides somewhat better agreement with the observations than does the simple equivalent thin screen model (the single uniformly extended medium model), if the refractive scintillation of the thin screen dominates the effect of the extended medium. The electron density fluctuation spectra along the line of sight to this pulsar agrees well with super-Kolmogorov spectra. In this way, we have provided an effective theoretical tool for investigating pulsar flux variations due to the refractive scintillation and for measuring the interstellar plasma properties and spatial dimensions of the Vela nebula more accurately.

The logarithmic slope of the structure function generally lies in the range $0.4<\gamma<1.4$ as mentioned by S2000, indicating that the scattering materials is not heavily concentrated in one or a few thin screens along the line of sight. There is evidence that different types of turbulent spectra exist in different regions of the Galaxy (Smirnova et al. 1998; Lambert \& Rickett 2000). A discontinuity spectrum $(\beta=4)$ is one way that the spectrum can be steepened, but the discontinuous scattering structure along the line of sight would also cause similar enhancement of refractive scintillation and would steepen the structure function of the flux density. Such enhancements are likely to be associated with the Vela supernova remnant. There is the possibility that an enhanced scattering region exists within the thin screen medium in the nebula. There has been no special discussion about the logarithmic slope of the structure function for the Vela pulsar in the former refractive scintillation studies, although it is highly sensitive to the detailed distribution of the scattering material. It can be seen, however, that the proposed two-component ISM model interprets the logarithmic slope of the structure function $\gamma=0.9$ to the Vela pulsar quite well.

Based on the above considerations, we adopt the $\beta=$ 4 model for the interstellar electron density fluctuations. However, neither the simple Kolmogorov spectrum nor the simple $\beta=4$ model is a universal model, so the exact form of the spectrum, especially the validity of a simple power-law description, is still a matter of research (Baht et al. 1999b). Since the two-component ISM model for $\beta=4$ provides reasonable agreement with observations, we propose that the distribution of density irregularities along the line of sight to the Vela pulsar follows a super-Kolmogorov form of power spectrum, which is consistent with the conclusions of S2000.

Multifrequency flux density structure function analyses of different pulsars are needed to investigate the real contribution of the thin screen medium and the extended medium to the refractive effects, and to determine the characteristic interstellar plasma turbulence parameters in different regions, such as the scattering strength and location of the thin screen medium. Our 
work may at least bring about some new results to pulsar refractive scintillation and ISM study by use of the two-component ISM model. We suggest that the line of sight passes through a region of enhanced density, which causes enhanced refractive scattering; this region may well have less small-scale substructure than in a turbulent medium and could include discontinuities. This might arise from refractive scattering effects caused in the Vela supernova remnant associated with the Vela pulsar.

Acknowledgements. We are grateful to the anonymous referee for valuable suggestions which improved the paper significantly. This work is supported by the National Natural Science Foundation (No. 10473018 and No. 10333020) in China.

\section{References}

Armstrong, J. W., Rickett, B. J., \& Spangler, S. R. 1995, ApJ, 443, 209

Aschenbach, B., Egger, R. J., \& Trümper, J. 1995, Nature, 373, 587

Bhat, N. D. R., Gupta, Y., \& Rao, A. P. 1998, ApJ, 500, 262

Bhat, N. D. R., Rao, A. P., \& Gupta, Y. 1999a, ApJ, 121, 483

Bhat, N. D. R., Gupta, Y., \& Rao, A. P. 1999b, ApJ, 514, 249

Bhat, N. D. R., Rao, A. P., \& Gupta, Y. 1999c, ApJ, 514, 272

Bhat, N. D. R., \& Gupta, Y. 2002, ApJ, 567, 342

Bhat, N. D. R., Cordes, J. M., Camilo, F., \& Nice, D. J. 2004, ApJ, 605,759

Blandford, R. D., \& Narayan, R. 1985, MNRAS, 213, 591

Blandford, R., Narayan, R., \& Romani, R. W. 1986, ApJ, 301, L53

Caraveo, P. A., De Luca, A., Mignani, R. P., \& Bignami, G. F. 2001, ApJ, 561, 930

Cha, A., Sembach, K. M., \& Danks, A. C. 1999, ApJ, 515, L25

Coles, W. A. 1988, Proceedings Radio wave Scattering in the Intersteller Medium, AIP Conf. Ser., 174, 163

Cordes, J. M., \& Lazio, T. J. W. 2002 [arXiv:astro-ph/0207156]

Cordes, J. M., \& Lazio, T. J. W. 2003 [arXiv:astro-ph/0301598]

Cordes, J. M., \& Rickett, B. J. 1998, ApJ, 507, 846
Cordes, J. M., Piderbetsky, A., \& Lovelace, R. V. E. 1986, ApJ, 310, 737

Cordes, J. M., Weisberg, J. M., Frail, D. A., et al. 1991, Nature, 354, 121

Esamdin, A., Wu X. J., \& Zhang, J. 2000, Ch. J. Astron. Astrophys., 24,275

Esamdin, A., Zhou, A. Z., \& Wu, X. J. 2004, A\&A, 425, 949

Gupta, Y., Rickett, B. J., \& Coles, W. A. 1993, ApJ, 403, 183

Gupta, Y., Rickett, B. J., \& Lyne, A. G. 1994, MNRAS, 269, 1035

Hewish, A., Bell, S. J., Pilkington, J. D. H., Scott, P. F., \& Collins, R. A. 1968, Nature, 217, 709

Hjellming, R. M., \& Narayan, R. 1986, ApJ, 310, 768

Kaspi, V., \& Stinebring, D. 1992, ApJ, 392, 530

LaBrecque, D. R., Rankin, J. M., \& Cordes, J. M. 1994, AJ, 108, 1854

Lambert, H. C., \& Rickett, B. J. 2000, ApJ, 531, 883

Lyne, A. G., Anderson, B., \& Salter, M. J. 1982, MNRAS, 201, 503

Qian, S. J., \& Zhang, X. Z. 1996, Acta Astron. Sinica, 37, 421

Rickett, B. J. 1990, ARA\&A, 28, 561

Rickett, B. J., Coles, W. A., \& Bourgois, G. 1984, A\&A, 134, 390

Rickett, B. J., \& Lyne, A. G. 1990, MNRAS, 244, 68

Romani, R. W., Narayan, R., \& Blandford, R. 1986, MNRAS, 220, 19

Shishov, V. I. 1993, Astron. Rep., 37, 378

Sieber, W. 1982, A\&A, 113, 311

Simrnova, T. V., Shishov, V. M., \& Stinebring, D. R. 1998, Astron. Rep., 42, 766

Stinebring, D. R., \& Condon, J. J. 1990, ApJ, 352, 207

Stinebring, D. R., Faison, M. D., \& Mckinnon, M. M. 1996, ApJ, 460,460

Stinebring, D. R., Smirnova, T. V., Hankins, T. H., et al. 2000, ApJ, 539, 300 (S2000)

Taylor, J. H., \& Cordes, J. M. 1993, ApJ, 411, 674 (TC93)

Wang, N., Wu, X. J. Manchester, R. N., et al. 2001, CJA\&A, Vol. 1, No. 5,430

Wu, X. J., \& Chian, A. C. L. 1995, ApJ, 443, 261

Zhou, A. Z., Wu, X. J., \& Esamdin, A. 2003a, A\&A, 403, 1059

Zhou, A. Z., Wu, X. J., \& Esamdin, A. 2003b, Chin. Phys. Lett., 20, 1405

Zhou, A. Z., Esamdin, A., \& Wu, X. J. 2003c, Acta Astron. Sinica, 44 254 